\title{
Introduction to the Special Section on Advances in Causal Discovery and Inference
}

Identification of cause and effect is the ultimate goal for most scientific and social discoveries. Controlled experiments are an effective approach to such discoveries, but they are expensive and sometimes infeasible to conduct. With the advent of big data availability in many areas, finding causal relationships using automated procedures is increasingly possible. With its focus on this challenge, causal discovery and inference is now a fast growing area in machine learning.

Graphical causal models, the potential outcome model, and structural equation models are the three major modelling approaches to representation of causal relations and identification of causal effects. They have achieved many successes in various applications. More importantly, the principles and insights of causal inference help to solve several challenging machine-learning problems, such as model explainability, transfer learning, domain adaptation, and lifelong learning [1].

However, causal discovery and inference faces many challenges in theory and practice. They need strong assumptions, some of which are not verifiable in data. There is a lack of ground truth data for real-world evaluation of causal discovery and inference methods. Some of the algorithms whose results have asymptotic theoretical guarantees are not scalable to large and/or highdimensional data. More research is still needed to solve fundamental problems in causal discovery and inference, such as structure learning, false discovery control, assessment of causal discoveries, hidden variables, and nonlinear and/or heterogeneous causal relationships. More real-world applications of causal discovery and inference are also vital.

Many workshops and symposia have been organized to meet the increasing research interests and demands in causal discovery and inference. Some associate editors of this special issue have organized four KDD Causal Discovery workshops, from 2016 to 2019. More than 10 other workshops and symposia have been organized in the same period, such as NeurIPS Workshop From "What If?" To "What Next?": Causal Inference and Machine Learning for Intelligent Decision Making in 2017; NeurIPS Workshop Machine Learning and Causal Inference for Improved Decision Making in 2019; UAI Workshop Causation: Foundation to Application, 2016; UAI Workshop Causality: Learning, Inference, and Decision-Making, 2017; and UAI Workshop on Causal Inference, 2018.

We edit this special issue to showcase the research achievements in the past few years since the previous special issue on the same topic in 2016 was published in this journal. This special issue collects seven articles that fall into two groups: fundamental problems and applications.

The five articles in the first group study the fundamental problems in causal discovery and inference and present novel solutions for false discovery control in structure learning, causal relationship detection in simulation models, causal structure search in the presence of latent confounders, the shortest causal path discovery by local search, and conditional independence test for causal structure learning.

Discovering causal relationships from observational data is a fundamental problem. Little research work has studied the strategies for controlling false discovery rates in causal structure learning. The article "Estimating and controlling the false discovery rate of the PC algorithm using edge-specific p-values," by E. Strobl, P. Spirtes, and S. Visweswaran, presents an extension

(C) 2019 Copyright held by the owner/author(s).

2157-6904/2019/11-ART45

https://doi.org/10.1145/3359995

ACM Transactions on Intelligent Systems and Technology, Vol. 10, No. 5, Article 45. Publication date: November 2019. 
of the PC algorithm, called PC-p, to learn causal DAGs with p-value by computing edge-specific $\mathrm{p}$-values and controlling the false discovery rates of the edges.

Central to explanatory simulation models is their capability to show not only what but also why particular events occur. Detecting causal relationships is helpful for such an explanation. The article "Detecting causal relationships in simulation models using intervention-based counterfactual analysis," by B. Herd and S. Miles, proposes an intervention-based causal analysis method to detect the causal relationships in simulation models. The core of the method is a formal model that maps basic causal assumptions to causal observations and allows for the identification of combinations of assumptions that have a negative impact on observability.

Causal structure search in the presence of latent confounders is an important and challenging issue. The article "Stable specification search in structural equation models with latent variables," by R. Rahmadi, P. Groot, and T. Heskes, aims to model causal relationships among latent variables that are indicated through observed proxies in light of stability analysis. In their previous studies, the authors introduced the stable specification search for cross-sectional data, an exploratory causal method that combines the concept of stability selection and multi-objective optimization to search for stable and parsimonious causal structures across the entire range of model complexities. In this article, they have extended their previous method to estimate linear causal relations between latent variables and reported interesting applications of the extended methods to various real-world data sets.

It is of great interest to know the shortest causal paths in practice. The article "Local learning approaches for finding effects of a specified cause and their causal paths," by Y. liu, Z. Cai, C. Liu, and Z. Geng, studies the problem of finding the shortest paths from a cause node to its effect nodes without knowing a global causal structure. The authors have presented theoretical analysis on the solutions by local structure learning and on the identifiability of such paths. They have proposed a practical algorithm which identifies the shortest paths faster than the global learning algorithms.

Conditional independence tests are an essential component in traditional constraint-based causal structure learning, and in recent years many studies have been conducted regarding how to make conditional independence tests more reliable. The article "Measuring conditional independence by independent residuals for causal discovery," by H. Zhang, S. Zhou, J. Guan, and J. Huan, attempts to convert conditional independence tests to the independence tests between regression residuals. In particular, considering variables $\mathrm{X}$ and $\mathrm{Y}$ and a set of variables $\mathrm{Z}$, the authors investigate the relationship between conditional independence between $\mathrm{X}$ and $\mathrm{Y}$ given $\mathrm{Z}$ and the independence between the regression residuals $\mathrm{X}-\mathrm{E}[\mathrm{X} \mid \mathrm{Z}]$ and $\mathrm{Y}-\mathrm{E}[\mathrm{Y} \mid \mathrm{Z}]$. They show that in particular cases the two cases are equivalent. The authors make use of this observation to simplify conditional independence tests and demonstrate an improvement on constraint-based causal discovery.

The remaining articles are devoted to the applications of graphical model learning, one on estimating causal effect in a genome-wide association study with hidden variables and the other on local causal discovery for feature selection.

Dealing with hidden common causes is of vital importance for causal inference in real-world applications. The article "Towards accounting for hidden common causes when inferring cause and effect from observational data," by D. Heckerman, tackles the challenge of accounting for hidden common causes in the context of genome-wide association studies. Using domain-knowledge of the causal structure and likely associations between the common causes, observed genomic markers, and target outcomes, the article demonstrates in simulated experiments that causal parameters can be recovered despite the existence of hidden confounders. This article serves both as an important demonstration of the care necessary when analysing data in the presence of hidden 
confounders and as an invitation to generalize the proposed method to apply beyond the specific structure of genome-wide association studies.

The discovery of the Markov blanket for feature selection is a fundamental problem in machine learning since the Markov blanket of the class attribute is considered the optimal feature set for prediction. The article "BAMB: A balanced Markov Blanket discovery approach to feature selection" by Z. Ling, K. Yu, H. Wang, L. Liu, W. Ding, and X. Wu, proposes a new Markov Blanket discovery algorithm for balancing the efficiency of the search procedure and the accuracy of the search results, by finding the parents, children, and spouses and removing false positives from the candidate set. The results on 10 real-world data sets are reported to illustrate the superior behaviour of the proposed approach.

This special issue features a small sample of a large body of works that represents the state-ofthe-art research on the topic. We hope that this collection will motivate further research in the exciting area of causal discovery and inference.

Jiuyong Li

University of South Australia, Australia

Kun Zhang

Carnegie Mellon University, USA

Emre Kıcıman, Microsoft Research, USA

Peng Cui

Tsinghua University, China

27 August 2019

\section{REFERENCE}

[1] J. Pearl. 2019. The seven tools of causal inference, with reflections on machine learning. Commun. ACM, 62, 3 (2019), $54-60$. 